\title{
Research on new energy utilization based on cloud computing and big data
}

\author{
Liang GAO ${ }^{1}$, Xinyan ZHANG ${ }^{1,2}$, Tao TONG ${ }^{1}$, Jiajun ZHANG ${ }^{1}$ \\ ${ }^{1}$ College of Electric engineering, Xinjiang University, China \\ ${ }^{2}$ Engineering Research Center of Education Ministry for Renewable Energy Power \\ Generation and Grid Technology, Xinjiang University, China \\ 1793346200@qq.com, xjcxzxy@126.com , 1378104278@qq.com
}

Keywords:Big data; Cloud computing; new energy; solar energy; prediction

Abstract: Environmental, clean and sustainable new energy sources have been paid much attention by researchers all over the world due to the increasingly exhaustion of traditional energy resources and the worsening of the ecological environment. Wind and solar energy are typical new energy sources, they cannot "live in harmony" with the power grid because of wind and solar energy have great intermittent and volatility, which greatly reduced the utilization efficiency of new energy based on wind and solar energy. Initially, This paper analyzes the relationship between big data, cloud computing and new energy, and the development of large data, cloud computing and new energy; Then, parsing challenges of new energy big data; Last, through typical example in the new energy industry to realize the promoting effect of cloud computing and big data on new energy, proving the feasibility and efficiency of cloud computing and big data in the field of new energy.

\section{Introduction}

At present, the world is facing two major problems: energy and climate warming [1]. The traditional fossil energy represented by oil are increasingly exhausted, and the price is fluctuating, social development consumes a large amount of fossil fuel, which leads to the rapidly growth of greenhouse gas emissions, how to get rid of the dependence on fossil fuels and reduce the emission of greenhouse gases, which has become a difficult problem to be solved urgently. The new energy, represented by wind energy and solar energy, stands out with its clean, environmental and sustainable advantages, received extensive attention. However, it is unable to "live in harmony" with the power grid", causing serious abandonment in the wind and solar energy[2], reducing the utilization efficiency of new energy based on wind and solar energy, which is seriously inconsistent the China source strategy of green, environmental protection and sustainable development[3]. In twenty-first Century, with the concept of big data and cloud computing put forward, the" energy revolution" of mankind has been greatly impacted [4-6].

The new energy industry as the basis of the future sustainable development of the country, is facing challenges and problems that cannot be ignored. It is necessary to solve a series of problems in the development of energy through big data and cloud computing [7]. 


\section{New energy based on big data and cloud computing}

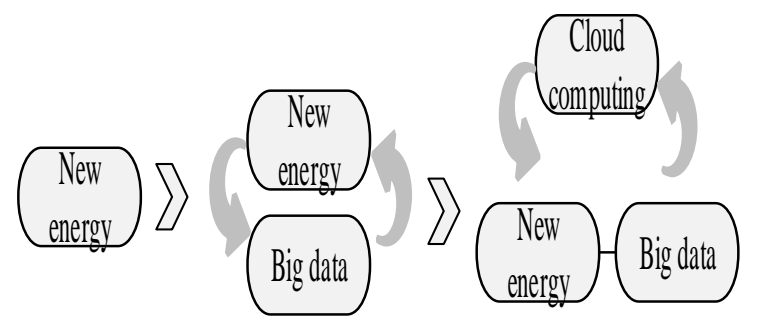

Fig.1 New energy data cloud computing relation diagram

"Big data", the size of the data involved is too large to use the current mainstream software tools, to achieve collection, analysis, processing or conversion to make decisions in a certain period of time.

"Cloud computing", based on Internet related services. The core of cloud computing is massive data storage and data parallel processing technology. Cloud computing provides available, convenient and on-demand Internet access.

"Big data" focuses on data, providing data collection, mining and analysis technologies. "Cloud computing" is mainly concerned with computing, focusing on IT architecture. Although some new energy technology tends to mature, the new energy industry is still in the growth period of the life development cycle, In particular, the new energy based on the wind and solar energy, cannot be used efficiently due to their own volatility and intermittence.

In China, Da tang Qinghai Energy Development Co., Ltd. has signed an agreement with the state power grid Qinghai electric power company to become a power generation enterprise in the new energy data Innovation Park of the national Power Grid Corp system.

\section{Big data of new energy based on Cloud Computing}

\section{Big data networks}

The new energy is a part of the smart grid. In order to realize the influence of cloud computing and large data on the development of new energy, this paper will take some new energy main wind and solar energy from the smart grid as an independent model.

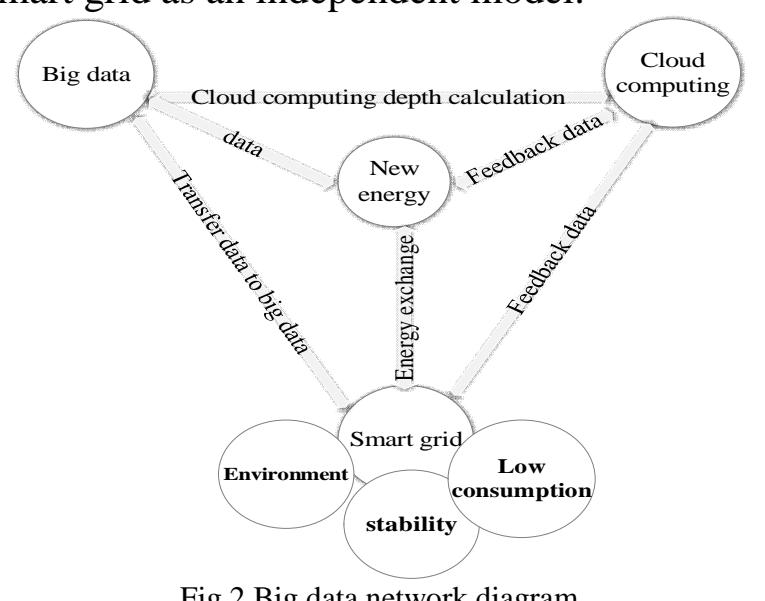

Fig.2 Big data network diagram

As shown in the Fig.2, the new energy industry and the power grid all need to transfer data information to big databases, big databases are connected with cloud computing, and the processed data are transferred to cloud computing. Under the conditions of environmental, stability and low consumption, cloud computing will feedback calculate information to the grid and new energy, can make new energy "live in harmony" with grid, improve the use efficiency of new energy [8].

\section{Big data technology challenge}

The new energy big data has the characteristics of large scale, multiple types, low value density and fast processing.

Cloud computing poses new challenges to big data, which is mainly reflected in storage, processing and analysis [9]. The traditional relational database which cannot meet the needs of big 
data processing. Large data has complex relational networks and complexity is not only reflected in the diversity of data types and the universality of data sources, but also in the uncertainty of distribution space and time. The value contained in big data needs to be deeply excavated, it is difficult to reduce the complexity of the algorithm, and a lot of traditional algorithms that cannot be applied in the big data environment.

\section{Application of big data and cloud computing in new energy field}

Using of big data has been integrated into the new energy a long time ago, with addition of cloud computing, the whole data structure becomes more complex and huge.

\section{Solar energy prediction}

At present, the common solar forecasting methods are divided into three kinds: the first is to predict the meteorological data directly as the input of the model; the second is to predict the historical observation data directly as the input of the model, and the third is to use the meteorological parameters and the historical observation data as the input of the model.

Traditional predictions use historical observation data and a few meteorological parameters, the prediction accuracy not meet the requirements. With development of big data, range of knowledge involved in solar prediction is broad.

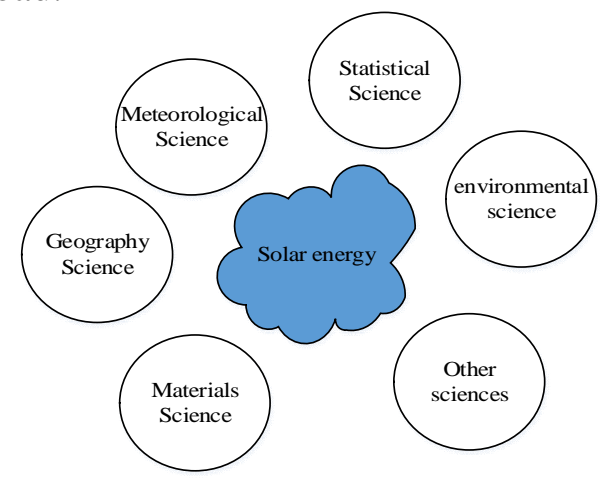

Fig.3 Solar energy related fields

\section{solar energy forecasting process}

Collecting solar energy related historical data, processing data simply, and finally calculating and analyzing potential data by algorithm. Achieve the purpose of accurate prediction of solar energy, solar energy will be used effectively and rationally.

1) Data collection

Collect all data information related to solar energy related disciplines.

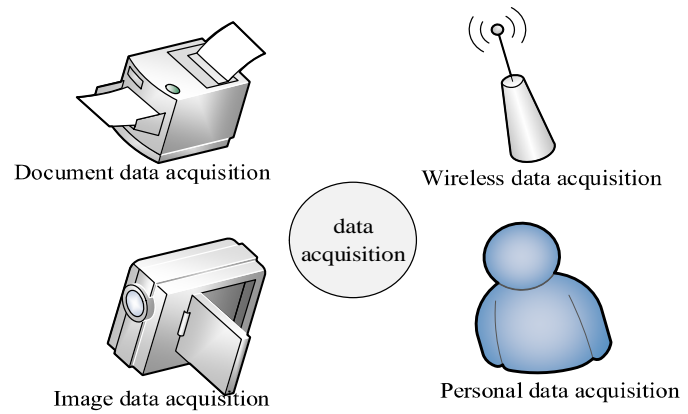

Fig.4 data acquisition 
2) Data processing

Processing the original measurement data to ensure the correctness and reasonableness of data.

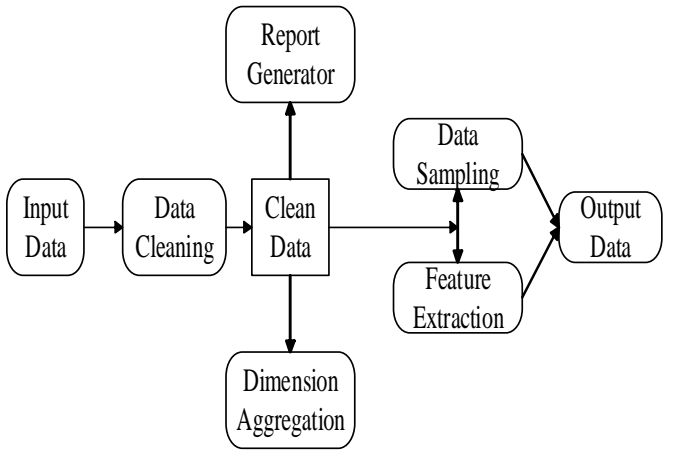

Fig.5 Data processing flow chart

3) Data analysis

Using cloud computing to analyze data and discover valuable information.

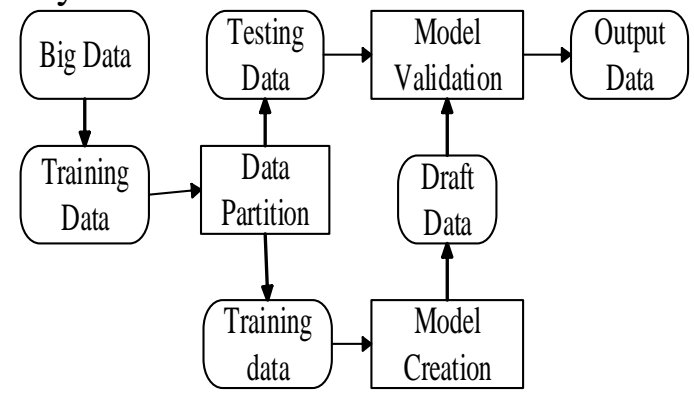

Fig.6 Flow chart of cloud computing data analysis

\section{Analysis of prediction results}

Through the historical solar radiation and meteorological data in $\mathrm{Ha} \mathrm{mi}$ area (data source: NSNA), Predict the solar radiation of the first 100 days of the year, prediction results as Fig.7.

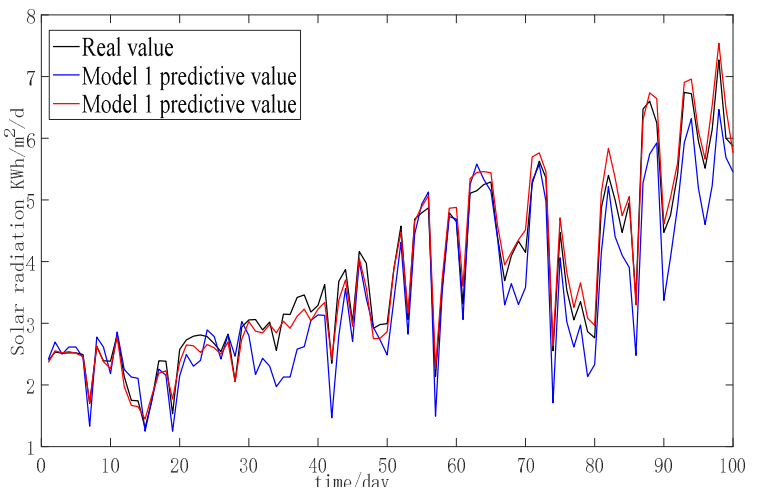

Fig.7 Prediction curve of solar radiation

The black curve is the actual value, red curve and blue curve are two different forecasting methods. It is clear to see that the prediction effect is basically the same as the actual value, and has a high precision. Using big data and cloud computing to predict solar energy will improve the efficiency of solar energy utilization.

\section{Conclusion}

Big data and cloud computing are widely applied in various fields, opportunities and challenges coexist, there are still many problems to be improved. New energy industry will be the mainstream of future energy structure, big data and cloud computing have great potential in new energy. Although there are many obstacles in the use of new energy, with the development of Internet computer, Reasonable combination of Internet big data, cloud computing and new energy will improve the current new energy problem that new energy cannot efficiently integrate into the power grid. 


\section{Acknowledgements}

This research was financially supported by National Natural Science Foundation of China (Grant No.51667018\&No.51367015).

\section{References}

[1] China Meteorological Administration. People's Republic of China meteorological industry standard-solar resource evaluation method. [S], 2007.

[2] Gable H. Grid-connected photo voltaic [J]. Solar Energy, 2001, 70(6): 455-456

[3] Zheng Zhijie, Li Lei. Discussion on some issues of large-scale photovoltaic grid connected power station access system [J]. Power grid and clean energy.2010，26(2):74-76

[4] D. Luo, C. Ding and H. Huang. Parallelization with Multiplicative Algorithms for Big Data Mining.IEEE 12th International Conference on Data Mining.

[5] K. Shim. MapR educe algorithms for big data analysis, and storage of big data, In Proceedings of the VLDB Endow ment, Istanbul, Turkey, pages 2016-2017. 2012.

[6] Junbo Zhang, Tianrui Liang, Yi Pan ,Parallel Rough Set Based Knowledge Acquisition Using MapR educe from Big Data, Big Mine'12, pages:20-27,2012.

[7] Kang Liyan, Zhang Ye, Cai Yingkai. Application and development of power user electric energy data acquisition system to smart grid [J] . Northeast Electric Power Technology, 2013 (7): 50-52.

[8] Hong Jianguang, Wu Kaifeng, Pei Xubin. Research and application of cloud computing based key technologies of performance improvement in electricity consumption information collection system [J] .Electric Power ICT, 2014, 12: 1-4.

[9] McKinsey Global Institute Big data: the next frontier for innovation, competition, and productivity[R]. 2011 